\title{
Hepatic nNOS impaired hepatic insulin sensitivity through the activation of p38 MAPK
}

\author{
Tianxue Zhao*, Qian Li”, Qianyun Mao, Kaida Mu and Chen Wang \\ Department of Endocrinology and Metabolism, Shanghai Key Laboratory of Diabetes Mellitus, Shanghai Diabetes Institute, Shanghai Jiao Tong University \\ Affiliated Sixth People's Hospital, Shanghai, People's Republic of China
}

Correspondence should be addressed to C Wang: wangchen@sjtu.edu.cn

*(T Zhao and Q Li contributed equally to this work)

\begin{abstract}
Neuronal nitric oxide synthase (nNOS) interacts with its adaptor protein NOS1AP through its PZD domain in the neurons. Previously, we had reported that NOS1AP enhanced hepatic insulin sensitivity through its PZD-binding domain, which suggested that nNOS might mediate the effect of NOS1AP. This study aimed to examine the role and underlying mechanisms of nNOS in regulating hepatic insulin sensitivity. nNOS co-localized with NOS1AP in mouse liver. The overexpression of NOS1AP in mouse liver decreased the level of phosphorylated nNOS (p-nNOS (Ser1417)), the active form of nNOS. Conversely, the liver-specific deletion of NOS1AP increased the level of p-nNOS (Ser1417). The overexpression of nNOS in the liver of high-fat diet-induced obese mice exacerbated glucose intolerance, enhanced intrahepatic lipid accumulation, decreased glycogen storage, and blunted insulin-induced phosphorylation of IRbeta and Akt in the liver. Similarly, nNOS overexpression increased triglyceride production, decreased glucose utilization, and downregulated insulin-induced expression of p-IRbeta, p-Akt, and p-GSK3beta in the HepG2 cells. In contrast, treatment with N $\omega$-propyl-L-arginine (L-NPA), a selective nNOS inhibitor, improved glucose tolerance and upregulated insulininduced phosphorylation of IRbeta and Akt in the liver of ob/ob mice. Furthermore, overexpression of nNOS increased p38MAPK phosphorylation in the HepG2 cells. In contrast, inhibition of p38MAPK with SB203580 significantly reversed the nNOS-induced inhibition of insulin-signaling activity (all $P<0.05$ ). This indicated that hepatic nNOS inhibited the insulin-signaling pathway through the activation of p38MAPK. These findings suggest that nNOS is involved in the development of hepatic insulin resistance and that nNOS might be a potential therapeutic target for diabetes.
\end{abstract}

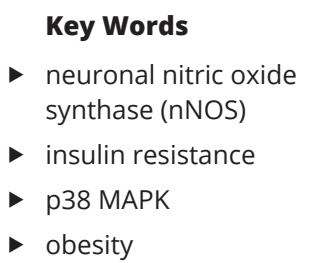

Journal of Endocrinology (2021) 248, 265-275

\section{Introduction}

Non-alcoholic fatty liver disease (NAFLD), a common chronic liver disease, manifested as hepatic steatosis and insulin resistance. The pathogenesis of NAFLD has many similarities with type 2 diabetes (T2D), and might underline the basis of the development of T2D (Loria et al. 2013). However, the mechanisms underlying NAFLD development have not been elucidated. Currently, there is a need to identify effective therapeutic targets for NAFLD.

Nitricoxidesynthase(NOS) is an enzymethat catalyzes nitric oxide (NO) synthesis. Excessive production of 
NO decreases glycogen synthase activity and increases glucose production in the liver (Borgs et al. 1996, An et al. 2008, Sansbury \& Hill 2014). Inducible NOS (iNOS), endothelial NOS (eNOS) and neuronal NOS (nNOS) (also called NOS1) are the three isoforms of NOS. The liverspecific overexpression of iNOS induced hepatic insulin resistant through S-nitrosylation of Akt (Shinozaki et al. 2011, Yang et al. 2015), whereas, the inhibition of iNOS reversed hyperglycemia and elevated insulin sensitivity in the liver of genetic obese mice (Fujimoto et al. 2005). eNOS, highly expressed in endothelial cells, played a role in regulation of vasorelaxation through the activation of insulin-signaling pathway (Muniyappa et al. 2007). The animal models of NAFLD are associated with liver endothelial cell dysfunction (Tateya et al. 2011), which resulted in the downregulation of eNOS activity and exacerbation of hepatic steatosis, fibrosis, and insulin resistance under obese conditions (Pasarin et al. 2011, Sheldon et al. 2014). nNOS is highly expressed in neurons and skeletal muscle, and moderate expressed in heart and pancreas (Bachar et al. 2010, Mezghenna et al. 2011, Lu et al. 2013, Baldelli et al. 2014). While the inhibition effect of iNOS on hepatic insulin sensitivity and the activation effect of eNOS on endothelial insulin sensitivity are well-documented, the reported role of nNOS in the regulation of insulin activity is controversial at the present. Mezghenna et al. reported that nNOS expression decreased in skeletal muscle of obese Zucker $(\mathrm{fa} / \mathrm{fa})$ rats, overexpression of nNOS promoted insulinstimulated Glut4 translocation and glucose uptake in the muscle cells (Mezghenna et al. 2014). However, lipid infusion-induced activation of hypothalamus nNOS resulted in enhanced hepatic glucose production (Marsollier et al. 2009). The exact role of nNOS within the hepatocytes is not clear. Recently, we demonstrated that NOS1 adaptor protein (NOS1AP) regulates hepatic insulin sensitivity through its PZD-binding domain (Mu et al. 2019). Several proteins, including nNOS, are reported to bind to the PZD-binding domain of NOS1AP (Jaffrey et al. 1998, Zhu et al. 2014). According to Jaffrey's reports, the binding of NOS1AP to nNOS in neurons diminished nNOS activity (Jaffrey et al. 1998). While previous study has revealed the dependency of NOS1AP on its PZD-binding domain on insulinsensitizing effect in liver of genetic and diet-induced obese (DIO) mice, more work is required to explore the role of nNOS in the regulation of insulin activity in liver, and the underlying mechanisms.

\section{Materials and methods}

\section{Recombinant adenoviruses, materials and antibodies}

Recombinant adenoviruses encoding nNOS (Ad-nNOS) and control virus (EGFP) were generated using the Gateway system (Invitrogen). Briefly, nNOS with HA as a tag was constructed into adenoviral plasmids (Invitrogen). Multiplicity of infection (MOI) of the generated adenoviruses was determined by an end-point dilution assay. Palmitate $(\mathrm{PA})$ and oleate $(\mathrm{OA})$ were purchased from Sigma. N $\omega$-PropylL-arginine (L-NPA) was from Cayman Chemical (Ann Arbor, MI, USA). SB203580 was purchased from MedChem Express (New Jersey, USA). Protein A/G agarose beads were from GE Healthcare. The antibodies used in this study were obtained from Cell Signaling Technology (rabbit anti-tubulin, antibeta-actin, anti-IR, anti-phospho-IR (Tyr1150/1151), antiGSK3beta, anti-phospho-GSK3beta (Ser9), anti-phospho-Akt (Ser473) antibodies, anti-p38 mitogen-activated protein kinase (MAPK), anti-phospho-p38 MAPK (Thr180/Tyr182), anti-PPARgamma, anti-FoxO1 and anti-PCK1), Novus Biologicals (rabbit anti-DGAT1), Sigma (rabbit anti-phosphonNOS/NOS1(Ser1417)), Abcam (rabbit anti-phospho-nNOS (Ser1417), anti-nNOS and -iNOS antibodies), Santa Cruz (anti-NOS1AP) and KangChen Bio-tech Inc. (Shanghai, China) (Mouse anti-GAPDH).

\section{Animal studies}

The ob/ob and $\mathrm{db} / \mathrm{db}$ mice which were purchased from Shanghai Laboratory Animal Center (Shanghai, China) were fed with a chow diet. The C57BL/6 male mice (aged 6 weeks) were obtained from Shanghai Slaccas Company (Shanghai, China) and fed either with chow diet or with a high-fat diet (HFD: 20\% kcal protein, 60\% kcal fat and $20 \%$ kcal carbohydrates; Research Diets, New Brunswick, USA) for 8 weeks. NOS1AP liver condition knockout and liver overexpression mice, and recombinant adenoviruses carrying NOS1AP were generated as previously reported (Mu et al. 2019). All the mice were housed at $23^{\circ} \mathrm{C} \pm 1^{\circ} \mathrm{C}$ with a $12 \mathrm{~h}$ light: $12 \mathrm{~h}$ darkness cycle and had free access to food and water. Procedures involving the care and use of animals were performed according to the Guidelines for the Care and Use of Laboratory Animals of Shanghai Jiao Tong University and approved by the Animal Ethics Committee of the University Affiliated Sixth People's Hospital (DWSY2011-096/DWLL2019-0274). To overexpression of nNOS in the liver, HFD- or chow dietfed C57BL/6 mice (aged 15 weeks for HFD and 10 weeks https://joe.bioscientifica.com https://doi.org/10.1530/JOE-20-0322 (c) 2021 Society for Endocrinology Published by Bioscientifica Ltd. Printed in Great Britain 
for chow diet-fed mice) were injected with Ad-nNOS (108 plaque-forming units) through the tail vein. Virus infection in liver of mice fed with chow diet had no obvious effect on mouse liver morphology (data not shown). Glucose tolerance test (GTT), insulin tolerance test (ITT), and insulin-stimulation assays were performed for 10-14 days post-transfection. To perform GTT, mice were fasted for 12 $\mathrm{h}$ and intraperitoneally injected with glucose at a dose of $1.5 \mathrm{~g} / \mathrm{kg}$ body weight. ITT was performed with mice of $6 \mathrm{~h}$ fasting and $1.5 \mathrm{U} / \mathrm{kg}$ insulin injection. For in vivo insulinstimulation assay, mice were fasted for $16 \mathrm{~h}$ and injected with $5 \mathrm{U} / \mathrm{kg}$ insulin intraperitoneally thereafter. The mice were sacrificed through cervical dislocation after $10 \mathrm{~min}$ and the liver was collected, frozen in liquid nitrogen and stored at $-80^{\circ} \mathrm{C}$. For in vivo nNOS inhibition, ob/ob mice (aged 8-9 weeks) or chow diet-fed C57BL/6 male mice (aged 10 weeks) were intraperitoneally injected with L-NPA $(20 \mathrm{mg} / \mathrm{kg})$, a nNOS specific inhibitor. After $2 \mathrm{~h}$, GTT or in vivo insulin-stimulation assay were performed.

\section{Human samples}

Human liver tissue was collected from patients of benign focal hepatic lesions undergoing liver surgery at the Department of Liver Surgery (Zhongshan Hospital, Fudan University, Shanghai, China) (Li et al. 2010). The protocol was approved by the Ethics Committee of Shanghai Jiao Tong University (2018-KY-026 (K)). Informed consent was given by all subjects and principles of the Declaration of Helsinki were followed.

\section{Cell culture and treatments}

The HepG2 (a kind gift from Dr F Liu, University of Texas Health Science Center at San Antonio, TX, USA from ATCC) was cultured in DMEM (G25) (Gibco) (Dong et al. 2015). The overexpression of nNOS in the HepG2 cells was achieved by infecting the cells with 10 multiplicity of infection (MOI) of Ad-nNOS or EGFP (control) for $16 \mathrm{~h}$. The virus-containing medium was, then, removed, and cells were cultured for additional $24 \mathrm{~h}$. To detect triglyceride production, the Ad-nNOS transfected HepG2 cells were treated with $0.4 \mathrm{mM}$ $\mathrm{PA}$ in combination with OA or $1 \%$ BSA for $24 \mathrm{~h}$. The insulinstimulation experiment was performed by culturing the Ad-nNOS transfected HepG2 cells in the presence or absence of $20 \mu \mathrm{M} \mathrm{SB} 203580$ for $30 \mathrm{~min}$, followed by treatment with $100 \mathrm{nM}$ insulin for $30 \mathrm{~min}$. Glucose consumption assay was conducted by transfecting the HepG2 cells with 5, 10, 15 and 20 MOI of Ad-nNOS. The glucose concentration was measured using a glucose oxidase-peroxidase assay kit (Applygen, Beijing, China).

\section{Quantitative real-time PCR, Western blotting and co-immunoprecipitation (Co-IP)}

Liver or cell total RNA was obtained using TRIzol reagents (Invitrogen). Quantitative real-time PCR was performed using Roche LightCycler 96 (Roche Ltd.) with SYBR Green Real-time PCR Master Mix-Plus (Toyobo, Osaka, Japan) at the annealing temperature of $60^{\circ} \mathrm{C}$. The relative expression level of each sample was calibrated by the comparative $\mathrm{Ct}$ method, using GAPDH as an endogenous control. Primers for each target gene were examined (data not shown). For Western blotting, the tissue or cell samples were homogenized in ice-cold RIPA buffer containing protease and phosphatase inhibitors. Equal amounts of protein were subjected to SDS-PAGE ( $8 \%$ or $10 \%$ ), transferred to a nitrocellulose membrane. The targeted proteins were detected using specific antibodies. The images were captured with ImageQuant LAS 4000 mini (GE Healthcare Life Science). To perform Co-IP, mouse liver tissues overexpression of NOS1AP were lysed using RIPA buffer. NOS1AP in the tissue lysates was precipitated using the anti-NOS1AP antibody bound to protein A/G agarose beads. The beads were washed with RIPA buffer, boiled in loading buffer and subjected to denaturing SDS-PAGE.

\section{Histology study and triglyceride measurements}

Liver tissue was fixed in 4\% paraformaldehyde, embedded in paraffin wax and subjected to hematoxylin and eosin (H\&E) staining. The frozen liver sections were subjected to Oil Red O staining. For periodic acid-Schiff (PAS) staining, the liver sections were incubated in $0.5 \%$ periodic acid solution after deparaffinization and hydration. A Zeiss Axio-Imager Standard Microscope (Carl Zeiss) was used to acquire digital images. Triglycerides in HepG2 cells were extracted with NP40, while those in the tissue were extracted using a heptane isopropanol-tween mixture as previously described (Mu et al. 2019). Triglycerides were measured using a Triglyceride assay kit (Nanjing Jiancheng Bioengineering Institute, Nanjing, China).

\section{Statistical analysis}

The data are shown as means \pm s.E.M. Statistical differences between two groups of variables were determined using Student's t-test, and among multiple groups of variables were determined by one-way ANOVA, followed by Bonferroni's multiple comparison post-tests. The correlation between two groups was determined by Pearson test. $P$-value of less than 0.05 was considered statistically significant. 


\section{Results}

\section{Hepatic nNOS activity was associated with insulin resistance in ob/ob mice}

The expression levels of nNOS in different mouse tissues and human liver were examined. Figure $1 \mathrm{~A}$ and $\mathrm{B}$ show the mRNA and/or protein expression levels of nNOS in the mouse brain, muscle, liver, pancreas, and heart with strong nNOS production in brain and muscle. Additionally, nNOS expression was detectable in human liver (Fig. 1C). In neurons, the activity of nNOS was regulated through interaction with NOS1AP by its C-terminal domain. Next, the physical interaction between NOS1AP and nNOS was examined. As shown in Fig. 1D, NOS1AP co-localized with nNOS but not with iNOS in mouse liver. The liver-specific overexpression of NOS1AP in mouse liver lowered hepatic phosphorylation of nNOS at Ser1417, an active form of nNOS (Fig. 1E). In contrast, the liver-specific deletion of NOS1AP increased p-nNOS expression (Ser1417) (Fig. 1F). Moreover, p-nNOS was increased in livers of $\mathrm{db} / \mathrm{db}$ diabetic mice compared with that of chow diet C57BL/6 mice (Fig. $1 \mathrm{G})$. Treatment with L-NPA $(20 \mathrm{mg} / \mathrm{kg})$, a specific nNOS inhibitor, improved glucose tolerance in ob/ob mice (aged 8-9 weeks) (Fig. 1H). The area under the curve of GTT in the L-NPA-treated ob/ob mice was significantly lower than
A

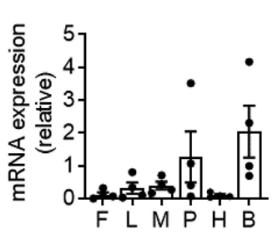

B

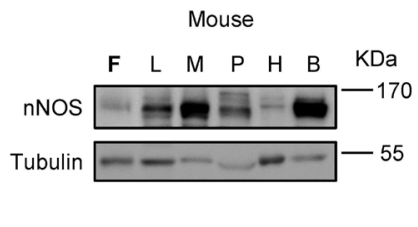

C

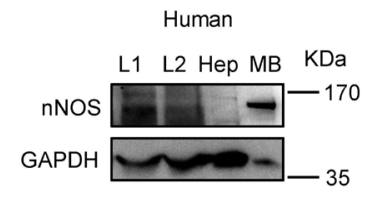

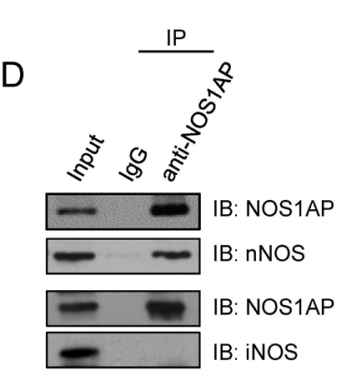

G

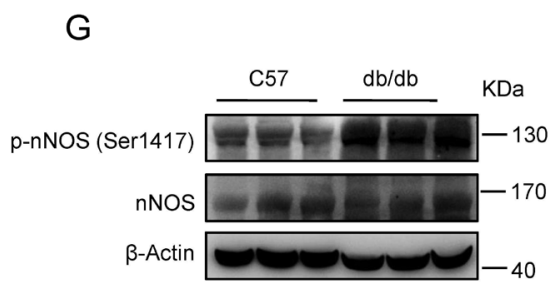

E
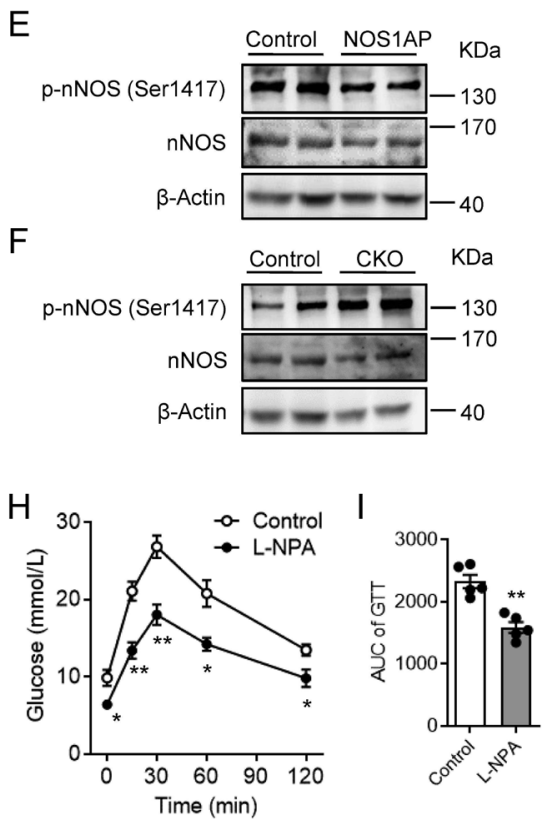

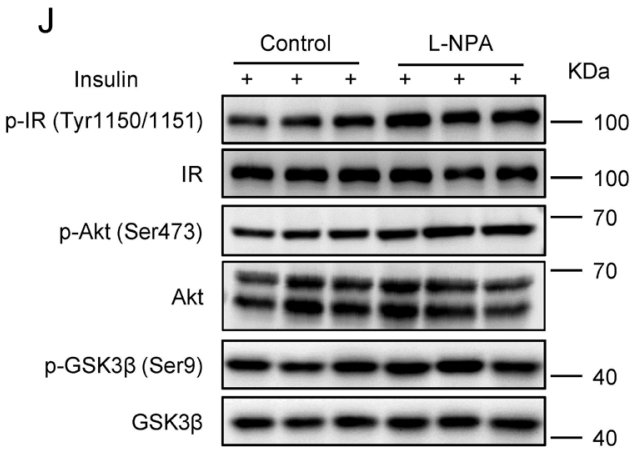

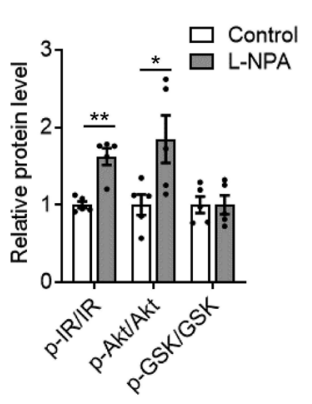

Figure 1

nNOS activity was associated with hepatic insulin resistance. (A and B) nNOS mRNA (A) and protein (B) expression levels in mouse tissues. $F$, fat; $L$, liver; $M$, muscle; $P$, pancreas; $H$, heart; $B$, brain. (C) Human liver nNOS protein expression. L1 and L2, human liver tissue; Hep, HepG2 cells; MB, mouse brain. (D) Co-localization of NOS1AP and nNOS in mouse liver. Mice were injected with adenovirus encoding NOS1AP. IB, immunoblot; iNOS, inducible nitric oxide synthase; IP, immunoprecipitation; NOS1AP, nitric oxide synthesis adaptor protein. ( $E$ and F) Western blotting of phosphorylation of nNOS at Ser1417 in mouse liver with NOS1AP overexpression (E) and NOS1AP deletion (CKO) (F). (G) Phosphorylation of nNOS at Ser1417 expression in mouse liver from C57BL/6 (C57) and db/db diabetic mice (aged 12 weeks). ( $\mathrm{H}$ and J) ob/ob mice (aged 8-9 weeks) were injected with L-NPA (nNOS specific inhibitor, $20 \mathrm{mg} / \mathrm{kg}$ ) or saline (control). Glucose tolerance test (GTT) was performed $2 \mathrm{~h}$ later $(\mathrm{H})$, and the AUC of GTT was calculated (I) $(n=5)$. () L-NPA treated ob/ob mice were injected with insulin (5 $\mathrm{U} / \mathrm{kg}$ ) for $10 \mathrm{~min}$. Western blot analysis was conducted to detect p-IR, p-Akt and p-GSK3 expression (left). Quantification of protein phosphorylation levels are normalized to total levels (right) $(n=5)$. Data represent means \pm S.E.M., $* P<0.05, * * P<0.01$ vs control (Student's $t$-test). 
that in the saline-treated ob/ob mice (Fig. 1I). No difference was observed in glucose tolerance between L-NPA-treated and saline-treated chow diet-fed C57BL/6 mice (data not shown). These findings indicated a correlation between nNOS activity and insulin resistance in the liver of obese mice. To confirm, phosphorylation of IR (Tyr1150/1151), AKT (Ser473) and GSK3beta (Ser9), three components of insulin-signaling, were detected, among which p-AKT is a key mediator of hepatocellular insulin action. As expected, treatment with L-NPA significantly enhanced the insulinstimulated expression of p-IR and p-Akt, but not that of p-GSK3beta, in ob/ob mice (Fig. 1J).

\section{Liver-specific overexpression of nNOS impaired glucose and insulin tolerance in HFD-induced obese mice}

To confirm the findings above, the adenoviral vectors encoding nNOS were generated and delivered to C57BL/6 mice fed with HFD for 8 weeks. The liver-specific overexpression of nNOS was confirmed using Western blotting (Fig. 2A). Overexpression of nNOS in mouse liver did not affect body weight and fasting blood glucose levels (Fig. 2B and C). However, injection of Ad-nNOS resulted in impaired glucose tolerance (Fig. 2D and E) and insulin sensitivity in the HFD-induced obese mice (Fig. $2 \mathrm{~F}$ and G). The areas under the GTT and ITT curve in mice transfected with adenovirus encoding nNOS was much higher than in control mice (Fig. 2E and G).

\section{Overexpression of nNOS exacerbated HFD-induced hepatic steatosis, impaired hepatic glycogen storage and HepG 2 cell glucose consumption}

The effect of nNOS overexpression on hepatic steatosis in the HFD-induced obesity mouse model was examined. Liver sections revealed more lipid droplets shown by $\mathrm{H} \& \mathrm{E}$ and Oil Red $\mathrm{O}$ staining after overexpression of nNOS (Fig. 3A and B). Consistently, hepatic triglyceride content significantly increased in the mice with nNOS overexpression (Fig. 3C). Moreover, the overexpression of nNOS increased the expression of mRNA and protein levels of Ppargamma and Dgat1 in liver of the HFD-fed obese mice (Fig. 3D and E). Similar phenomena were observed in HepG2 cells. Treatment with PA and OA significantly increased the triglyceride production in of the HepG2 cells. The overexpression of nNOS further enhanced triglyceride production in the presence of PA and OA (Fig. 3F).

Furthermore, nNOS overexpression decreased the glycogen storage in the liver of the HFD-induced obese mice, and glucose consumption in HepG2 cells (Fig. 3G and H). In consistency, the mRNA and protein expression levels of Pck1 and/or Foxo1 were upregulated in the liver upon nNOS overexpression (Fig. 3I and J). Similar phenomena were observed in HepG2 cells (data not shown). These data suggested a role of nNOS on hepatic glucose metabolism regulation in liver of obese mice.
A

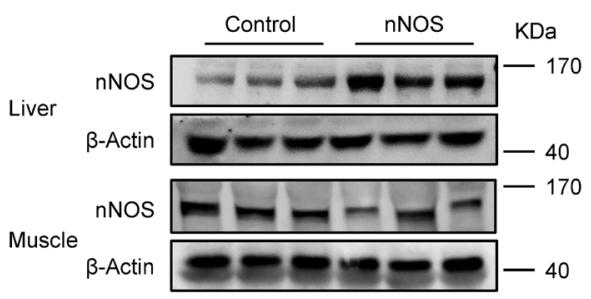

B
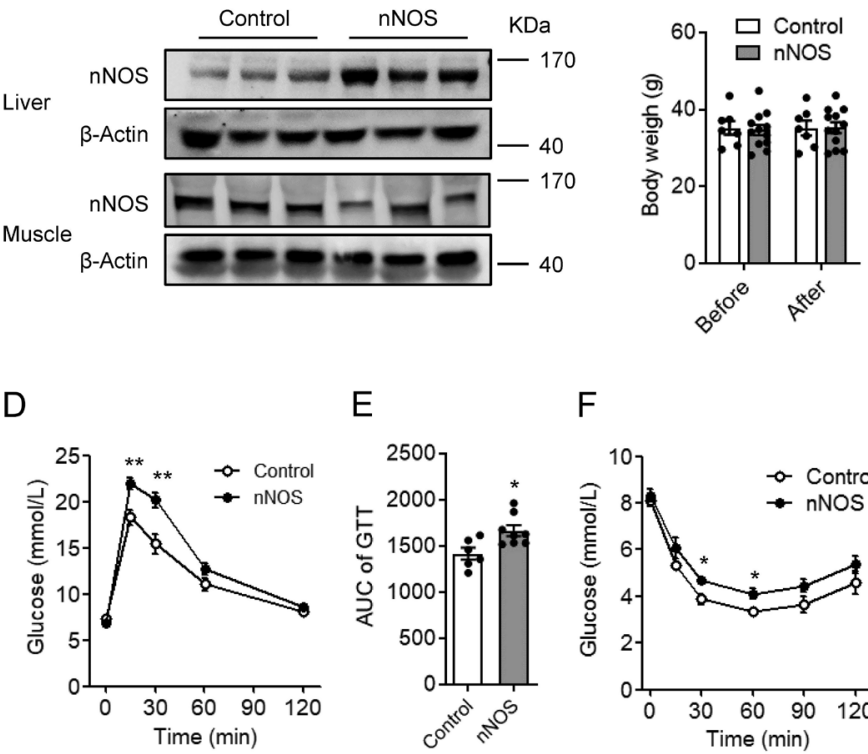

E

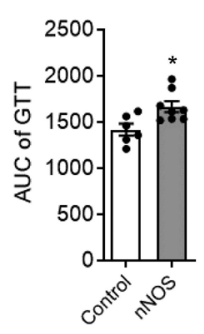

$\mathrm{F}$

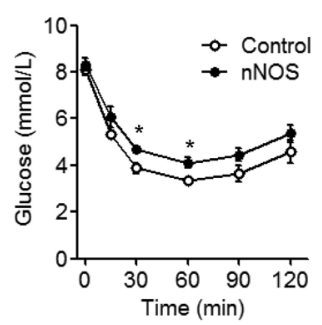

C

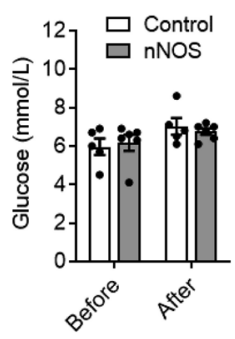

G

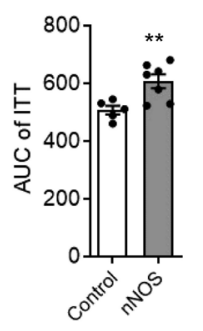

\section{Figure 2}

Overexpression of nNOS in liver impaired glucose and insulin tolerance of high-fat diet-induced obese mice. C57BL/6 mice were injected with adenoviruses encoding nNOS or EGFP (control) after 8 weeks of high-fat diet. (A) Western blot analysis of nNOS in liver. (B) Body weight ( $n=7-12)$ and (C) fasting blood glucose $(n=5-6)$ were detected before and after injection. ( $D$ and E) Glucose tolerance tests (GTT) $(n=6-8)$ and (F and $\mathrm{G}$ ) insulin tolerance tests (ITT) $(n=5-8)$ were conducted 9-12 days after injection, and the areas under curve (AUC) for GTT (E) and ITT (G) were calculated. Data represent means \pm S.E.M., $* P<0.05$ vs control (Student's t-test). 
A

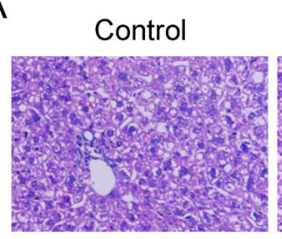

C

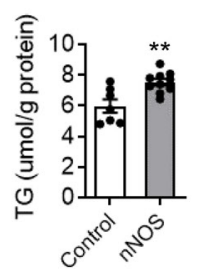

E
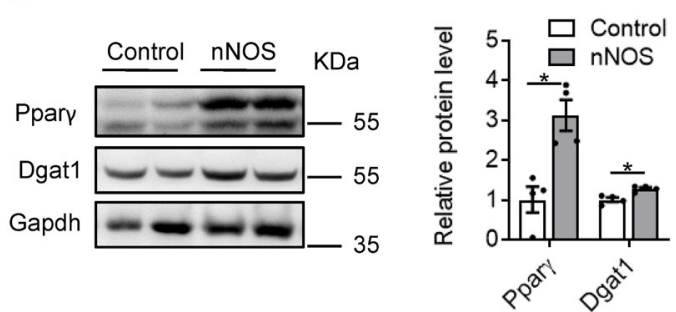

G
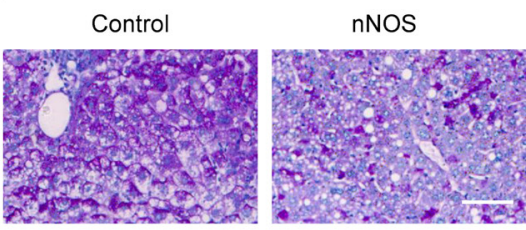

।

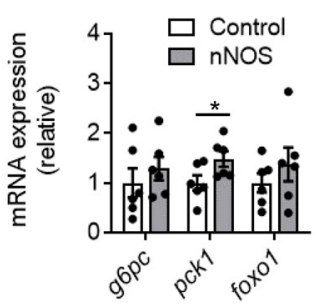

$J$

\section{Overexpression of nNOS inhibited insulin-signaling activity in HepG2 cells and mouse liver}

To confirm the effect of nNOS on hepatic insulin sensitivity, the activity of insulin-signaling pathway in hepatocytes with nNOS overexpression was examined in vitro and in vivo. Treatment with PA significantly lowered the levels of insulin-stimulated phosphorylation of IR (Tyr1150/1151), Akt (Ser473) and GSK3beta in HepG2 cells (Fig. 4A). The effect of PA on insulin signaling was mimicked by nNOS overexpression alone in the cells. Transfection with
$\mathrm{F}$

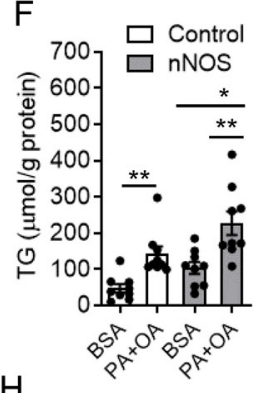

$\mathrm{H}$

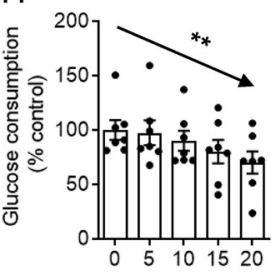

\section{Figure 3}

The overexpression of nNOS enhanced hepatocyte lipid accumulation, lowered glycogen storage and glucose consumption. C57BL/6 mice were injected with adenoviruses encoding $\mathrm{nNOS}$ (Ad-nNOS) after 8 weeks of high-fat diet (HFD). (A) $\mathrm{H} \& \mathrm{E}$ staining (scale bar, $100 \mu \mathrm{m}$ ) and (B) Oil Red O staining (scale bar, $25 \mu \mathrm{m}$ ) were conducted in liver of the HFD-fed mice. (C) Triglyceride (TG) content was detected in the liver of the mice $(n=7-10)$. (D) Real-time PCR and (E) Western blot analyses were performed on the liver extracts. Quantifications of mRNA $(n=5-6)$ and protein $(n=4)$ expression were normalized by Gapdh. Data represent means \pm S.E.M. ${ }^{*} p<0.05, * * p<0.01$ vs indicated groups (Student's t-test). HepG2 cells were exposed to Ad-nNOS. After $16 \mathrm{~h}$, the cells were treated with $0.4 \mathrm{mM}$ palmitate (PA) in combination with oleate (OA) for $24 \mathrm{~h}$. (F) TG content was detected from the HepG2 cells $(n=9)$. (G) PAS glycogen staining of the liver of the HFD-fed mice (scale bar, $100 \mu \mathrm{m}$ ). (H) The HepG2 cells were exposed to different concentrations of Ad-nNOS (5, 10, 15 and $20 \mathrm{MOI})$. Glucose consumptions of the cells were detected at the end of experiments. ${ }^{*} p<0.01$ for trend (Pearson test, $n=7)$. (I) Real-time PCR $(n=6)$ and (J) Western blot analyses were performed on liver extracts from HFD-fed mice, quantification of Western blotting was normalized by Gapdh $(n=4)$. Data represent means \pm S.E.M. ${ }^{*} P<0.05$ vs indicated groups (Student's t-test). cd36, fatty acid translocase; fas, fatty acid synthase; acc1, acetyl-CoA carboxylase1; scd1, stearoyl-Coenzyme A desaturase; dgat, diacylglycerol

O-acyltransferase; gpat, glycerol-3phosphateacyltransferase; $m t t p$, microsomal triglyceride transfer protein; $h s l$, hormonesensitive lipase; atgl, adipose triglyceride lipase; srebp1, Sterol-regulatory element binding proteins1; ppar, peroxisome proliferator activated receptor; acox1, acyl-Coenzyme A oxidase 1; g6pc, glucose-6-phosphatase; pck1, phosphoenolpyruvatecarboxykinase1; fox01, forkhead box protein 01.

Ad-nNOS significantly reduced insulin-induced expression of p-IRbeta, p-Akt and p-GSK3beta expression in the cells (Fig. 4A). Similar phenomena could be viewed in the mouse liver with nNOS overexpression (Fig. 4B).

\section{nNOS overexpression activated p38 MAPK signaling pathway in HepG2 cells}

Previous studies have reported that p38MAPK activation contributed to insulin resistance in liver and that nNOS regulates p38MAPK in neuronal PC12 cells. Then, the 
A
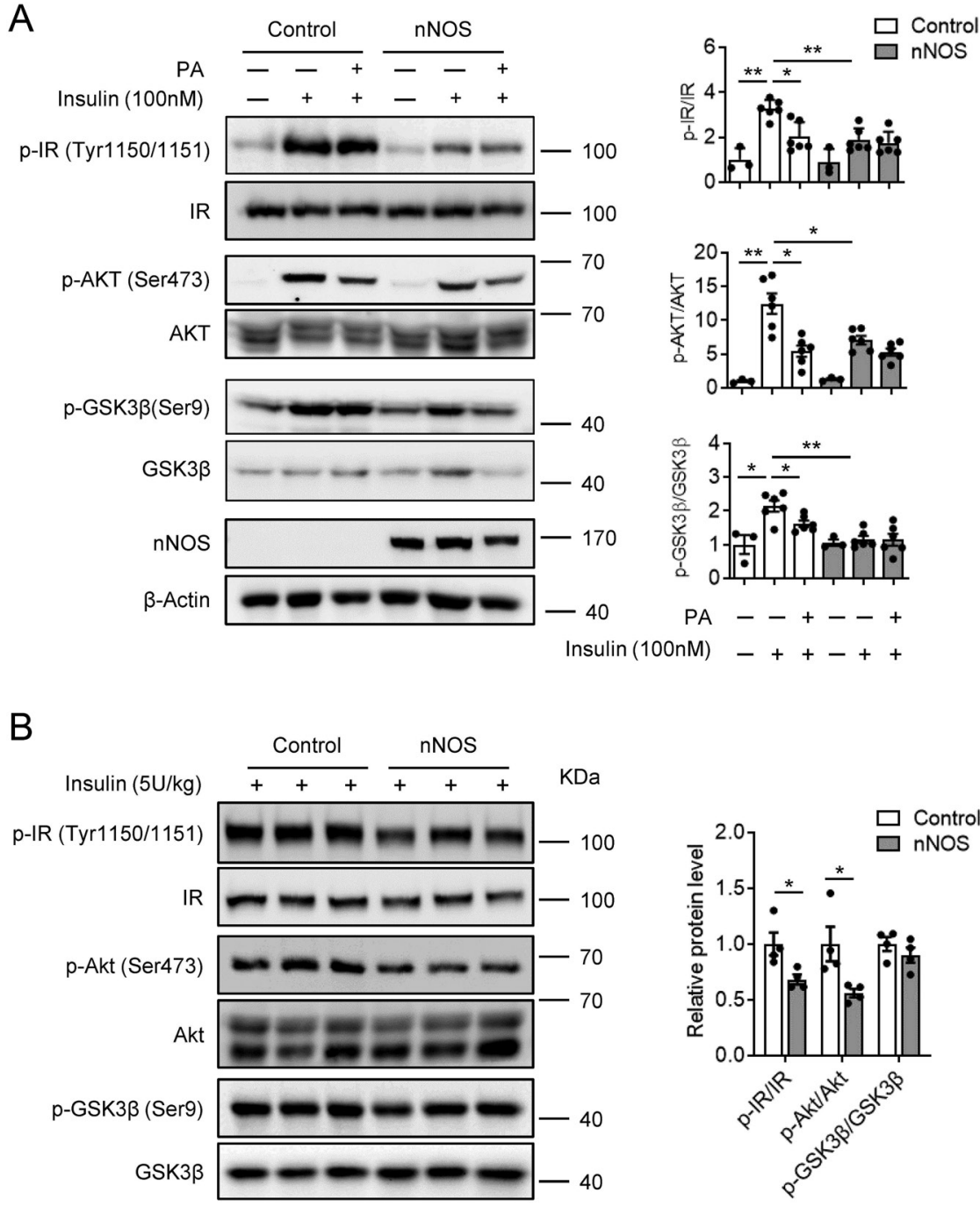

Figure 4

Overexpression of nNOS inhibited insulin-induced signaling activity in vitro and in vivo. (A) nNOS overexpressed HepG2 cells were first treated with palmitate (PA, $0.25 \mathrm{mM}$ ) for $24 \mathrm{~h}$. Then, the cells were exposed to $100 \mathrm{nM}$ insulin at the end of experiment for $30 \mathrm{~min}$. Data represent means \pm S.E.M., $n=3-6, * P<0.05, * \star P<0.01$ between indicated groups (One-way ANOVA with Bonferroni's multiple comparisontest). (B) C57BL/6 mice were injected with adenoviruses encoding nNOS. After 10 days, fasted mice were injected with insulin (5 U/kg) for $10 \mathrm{~min}(n=4)$. Western blot analysis was conducted to detect p-IR, p-Akt and p-GSK3beta expression (left). Quantification of protein phosphorylation levels are normalized to total levels (right). Data represent means \pm S.E.M., $* P<0.05$ $\star \star P<0.01$ (Student's t-test). effect of nNOS overexpression on p38MAPK activity in the hepatocytes was examined. As shown in Fig. 5, transfection with Ad-nNOS significantly increased the phosphorylation of p38MAPK and decreased insulinstimulated activation of insulin-signaling pathway. Inhibition of p38MAPK activity with its specific inhibitor SB203580 significantly lowered phosphorylation of p38MAPK, and mitigated the nNOS-induced inhibition of insulin signaling (Fig. 5). These results suggested that the inhibition effect of nNOS on insulin- sensitivity in HepG2 cells is dependent on the activation of p38MAPK.

\section{Discussion}

In the present study, we provide evidences demonstrating that nNOS played a role in inducing hepatic insulin resistance. The nNOS protein were detectable in human and mouse liver. The in vivo evidences revealed that the elevation of hepatic nNOS in obese mice impaired glucose tolerance, reduced hepatic glycogen storage, increased hepatic triglyceride content, and inhibited insulin-induced signaling activation in mouse liver and HepG2 cells. In contrast, inhibition of nNOS activity improved glucose tolerance and hepatic insulin sensitivity in ob/ob mice. Moreover, the inhibition effects of nNOS on insulin sensitivity was through the activation of p38MAPK in the HepG2 cells.

Hepatic insulin resistance contributes to the development of T2D. The findings of the present study established a link between the activation of nNOS and elevation of hepatic glucose production through the inhibition of insulin-induced signaling activity. nNOS activity was positively correlated with hepatic insulin resistance. Liver-specific nNOS overexpression exacerbated glucose and insulin intolerance in DIO mice. 

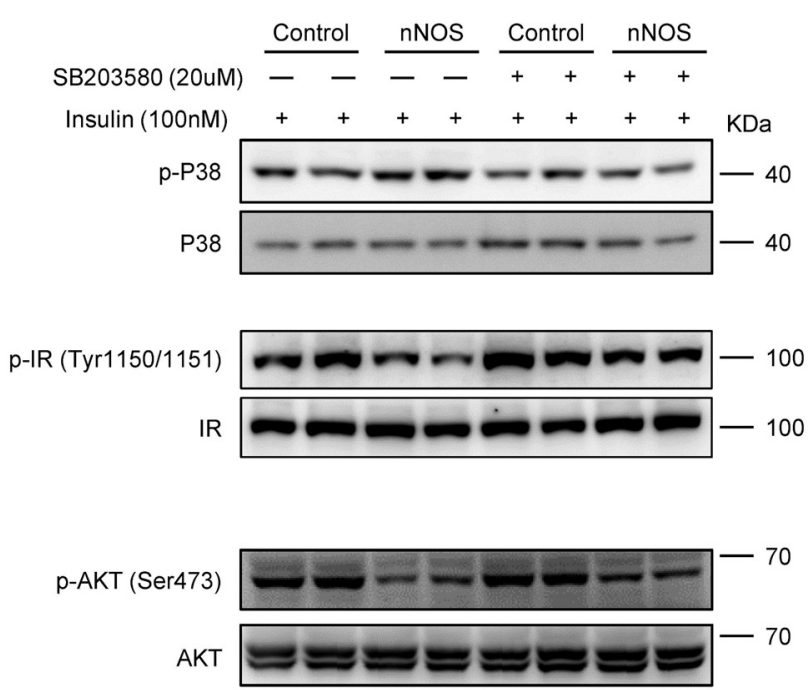

p-GSK3ß(Ser9)

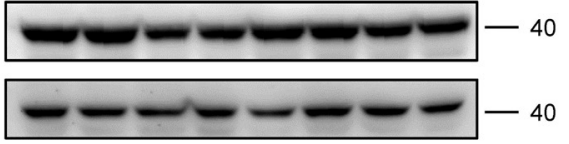

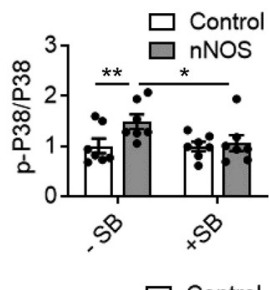
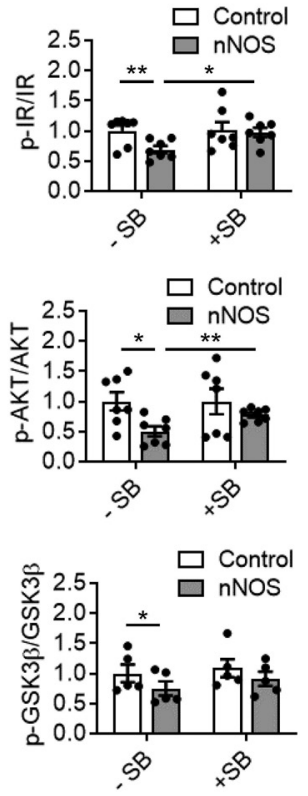

\section{Figure 5}

Overexpression of nNOS activated p38 MAPK activity. HepG2 cells were exposed to adenovirus encoding nNOS or EGFP (control) and treated with insulin (100 nM). (A) Western blot analysis of p-IR, $\mathrm{p}$-AKT and p-GSK3beta expression in the presence or absence of p38 MAPK inhibitor SB203580 (20 uM) for 30 min. (B) Quantification of protein phosphorylation levels are normalized to total levels $(n=5-7)$. Data represent means \pm S.E.M., $* P$ $<0.05$, ${ }^{*} P<0.01$ between indicated groups (One-way ANOVA with Bonferroni's multiple comparison test).
The overexpression of nNOS in liver reduced hepatic glycogen storage in mice and glucose utilization in the HepG2 cells. These changes were associated with upregulation of Pck1 and FoxO1 expression. PCK1 is a rate-limiting enzyme of gluconeogenesis in liver (Gomez-Valades et al. 2008). FOXO1, a transcription factor, mediates activation of PCK1 and downstream targets of insulin-signaling pathway (O-Sullivan et al. 2015). FoxO1-overexpressing mice exhibited impaired glucose tolerance and downregulated hepatic glycogen levels. In contrast, FoxO1 knockout mice exhibited enhanced glucose utilization and insulin sensitivity (Qu et al. 2006, Lu et al. 2012). Consistent with the findings of previous studies, this study demonstrated that nNOS overexpression upregulated the hepatic Foxo1 expression and downregulated the insulin-induced phosphorylation of proteins involved in the insulinsignaling pathway (IRbeta and Akt) both in vivo. Insulin exerts its effects by binding to its receptor, which promotes the autophosphorylation at the Tyr1150/1151 site of the insulin receptor beta subunits. The activation of insulin receptor, in turn, recruits and phosphorylates signaling of proteins, such as phosphoinositide-3-kinase/AKT, which are critical for hepatocyte insulin function. The downstream substrates of AKT include FOXO1 and GSK3beta. The activation of GSK3beta released the inhibition of glycogen synthase, which leads to the production of glycogen in liver (Petersen \& Shulman 2018). Glycogen can also be synthesized in the liver through a GSK3-independent pathway (Li et al. 2019). In contrast, inhibition of nNOS activity with L-NPA, a specific inhibitor, reversed glucose intolerance of ob/ob mice, and significantly improved insulin-stimulated hepatic signaling pathway activity in these mice. These findings suggested that nNOS exerts an inhibitory effect on the insulin-signaling pathway in the hepatocytes. The findings of the study were in consistent with these reported by Marsollier et al. (Marsollier et al. 2009) but not those reported by Shankar et al. (Shankar et al. 2000), and Nakata et al. (Nakata et al. 2008). Marsollier et al. reported that activation of hypothalamic nNOS activity leads to hepatic insulin resistance (Marsollier et al. 2009). Shankar et al. demonstrated that systemic nNOS knockout in mice resulted in mild insulin resistance (Shankar et al. 2000). Nakata et al. found normal glucose tolerance after systemic nNOS deletion (Nakata et al. 2008). The reasons for the contradictory results between different studies are unclear. It is worth noticing that in the present study the expression of nNOS in muscle tended to decrease following nNOS overexpression in the liver of obese mice (data not shown). Based on the findings from Mezghenna et al. that nNOS expression decreased in skeletal muscle of 
obese Zucker (fa/fa) rats (Mezghenna et al. 2014), the downregulation of nNOS in muscle might be secondary to the whole-body insulin resistance induced by liverspecific nNOS overexpression. It is expected that nNOS might play different roles on insulin sensitivity in different tissues. More work is needed to clarify the possibility. iNOS regulates hepatic insulin resistance through NO release and subsequent S-nitrosylation of the insulin-signaling pathway-related proteins (Carvalho-Filho et al. 2005, Shinozaki et al. 2011, Sansbury \& Hill 2014). In contrast to iNOS overexpression, the overexpression of nNOS did not result in the upregulation of NO production in the liver of obese mice (Data not shown). This suggested that the role of nNOS on insulin sensitivity inhibition in liver might be through NO-independent pathway. It is true that the activation of nNOS is not always associated with enhanced NO production (Sansbury et al. 2014).

Moreover, this study showed that nNOS is involved in the regulation of hepatic lipid accumulation, which is an early characteristic of NAFLD, in HFD-fed mice. In association with the increase in triglyceride accumulation upon nNOS overexpression in liver, the protein levels of Ppargamma and Dgat1 were significantly upregulated. DGAT1, a key enzyme in liver, catalyzes the terminal step of triacylglycerol synthesis. The expression of DGAT1 was significantly increased in patients with NAFLD (Kohjima et al. 2007). The overexpression of DGAT1 in mice resulted in the elevation of intrahepatic triglyceride synthesis (Millar et al. 2006). PPARgamma is a transcription factor that promotes lipid synthesis and regulates multiple enzymes involved in the uptake and synthesis of fatty acids (Gross et al. 2017). NAFLD is associated with the upregulated expression of PPARgamma (Moran-Salvador et al. 2011, Pettinelli \& Videla 2011). The knockout of PPARgamma decreased the expression of triglyceride synthesis-related enzymes DGAT1 and DGAT2 (Zhang et al. 2016), suggesting that hepatic nNOS might function as an aggravator for steatosis in obese mice. However, the mechanisms linking the expression of nNOS and PPARgamma are not clear.

Lastly, the results presented in the study demonstrated that the inhibitory effect of nNOS on insulin signaling was through the activation of p38 MAPK signaling in HepG2 cells. In the present study, the phosphorylation of p38MAPK was upregulated in the Ad-nNOS-transfected HepG2 cells, and the inhibition effect of nNOS on insulinsignaling pathway in HepG2 cells was significantly blunted when p38 MAPK activity was blocked with its specific inhibitor. This indicated that nNOS exerts an inhibitory effect on the insulin-signaling pathway in the HepG2 cells through p38MAPK. Previous studies have reported that
p38MAPK is activated in the liver of ob/ob or DIO mice (Gum et al. 2003, Sun et al. 2016). Activated p38MAPK inhibits the insulin-signaling pathway by downregulating the phosphorylation of insulin receptor substrate and AKT, which promotes insulin resistance. Moreover, activation of p38 MAPK participated in the regulation of liver glucose metabolism, promoted liver gluconeogenesis, and reduced glycogen synthesis and storage (Hemi et al. 2011). It is worth noticing that nNOS was reported to be the upstream mediator of phosphorylation of p38 MAPK induction and mediated excitotoxicity in the nervous system (Cao et al. 2005, Kajiwara et al. 2013, Lai et al. 2014). The findings of the present study that the inhibitory effect of nNOS on insulin signaling was dependent on p38MAPK were in line with the findings of previous studies on the neurons. The mechanisms underlying nNOS-mediated activation of p38MAPK in the HepG2 cells are unclear. In the nervous system, nNOS-mediated NO production enhances $\mathrm{Ca}^{2+}$ influx, which results in the activation of calmodulin kinase II and the phosphorylation of p38MAPK (Kajiwara et al. 2013, Lai et al. 2014). In this study, the overexpression of $\mathrm{nNOS}$ in the HepG2 cells or mouse liver did not significantly upregulate NO levels. This indicated that other factors were involved in nNOS overexpression-induced p38MAPK activation. Previously, we had reported that

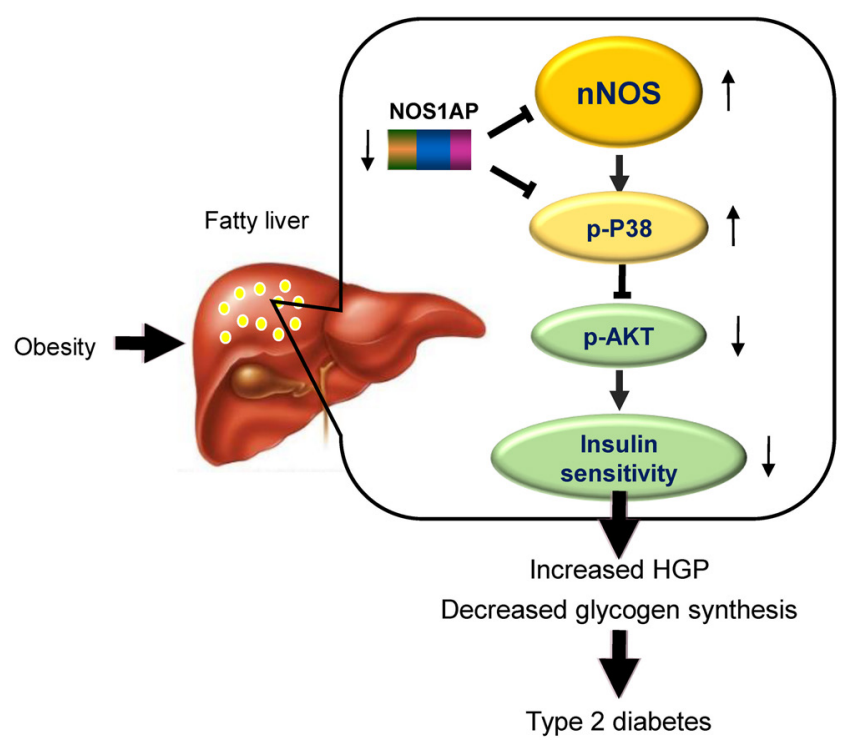

\section{Figure 6}

Regulatory effect of NOS1AP-nNOS on hepatic insulin sensitivity under obesity. Obesity induced triglyceride accumulation, and resulted in downregulation of NOS1AP activity and upregulation of nNOS activity in the liver. nNOS activation, in turn, promoted p38 MAPK activation and inhibited insulin-signaling pathway through the downregulation of the phosphorylation levels of Akt, which lead to increased hepatic glucose production (HGP), decreased glycogen synthesis and hepatic insulin resistance. 
the overexpression of NOS1AP enhanced hepatic insulin sensitivity and downregulated p-p38MAPK expression in the livers of ob/ob mice (Mu et al. 2019). In this study, the expression of NOS1AP in the liver was inversely correlated with that of nNOS active forms. The binding of NOS1AP to nNOS in the neurons promotes the activity of nNOS on its target protein. Hence, NOS1AP may exert its effect in the liver through the inactivation of nNOS.

Taking together, the findings of this study indicated that nNOS plays an inhibitory role in liver insulin sensitivity. The overexpression of nNOS promoted fat accumulation, decreased glycogen storage, and inhibited the insulin-stimulated signaling pathway in the liver of obese mice. Moreover, the overexpression of nNOS promoted p38 MAPK activation in HepG2 cells, which was essential for the inhibitory effects of nNOS on insulin signaling (Fig. 6). The inhibitory effects of nNOS on hepatic insulin-signaling activity highlight the importance of nNOS in the pathogenesis of NAFLD, and suggested that nNOS could be utilized as a potential therapeutic target to prevent diabetes by improving hepatic insulin sensitivity.

\section{Declaration of interest}

The authors declare that there is no conflict of interest that could be perceived as prejudicing the impartiality of the research reported.

\section{Funding}

This study was supported by the National Natural Science Foundation of China (81670707 to C. Wang). The funders had no role in the study design, data collection and analysis, decision for publish, or preparation of the manuscript.

\section{Author contribution statement}

$\mathrm{TZ}, \mathrm{QL}, \mathrm{QM}$ and $\mathrm{KM}$ contributed to experiment performance, and data analysis. TZ contributed to the manuscript drafting. CW designed the research, analyzed and interpreted the data, and critically revised the manuscript for important intellectual content, and is the guarantor of this work and, as such, had full access to all the data in the study and takes responsibility for the integrity of the data and the accuracy of the data analysis. All authors revised and approved the final version of the manuscript.

\section{Acknowledgements}

The authors thank Hui Zhu for technique support.

\section{References}

An Z, DiCostanzo CA, Moore MC, Edgerton DS, Dardevet DP, Neal DW \& Cherrington AD 2008 Effects of the nitric oxide donor SIN-1 on net hepatic glucose uptake in the conscious dog. American Journal of Physiology. Endocrinology and Metabolism 294 E300-E306. (https://doi. org/10.1152/ajpendo.00380.2007)
Bachar E, Ariav Y, Cerasi E, Kaiser N \& Leibowitz G 2010 Neuronal nitric oxide synthase protects the pancreatic beta cell from glucolipotoxicity-induced endoplasmic reticulum stress and apoptosis. Diabetologia 53 2177-2187. (https://doi.org/10.1007/ s00125-010-1833-6)

Baldelli S, LettieriBarbato D, Tatulli G, Aquilano K \& Ciriolo MR 2014 The role of nNOS and PGC-1alpha in skeletal muscle cells. Journal of Cell Science 127 4813-4820. (https://doi.org/10.1242/jcs.154229)

Borgs M, Bollen M, Keppens S, Yap SH, Stalmans W \& Vanstapel F 1996 Modulation of basal hepatic glycogenolysis by nitric oxide. Hepatology 23 1564-1571. (https://doi.org/10.1002/hep.510230637)

Cao J, Viholainen JI, Dart C, Warwick HK, Leyland ML \& Courtney MJ 2005 The PSD95-nNOS interface: a target for inhibition of excitotoxic p38 stress-activated protein kinase activation and cell death. Journal of Cell Biology 168 117-126. (https://doi.org/10.1083/jcb.200407024)

Carvalho-Filho MA, Ueno M, Hirabara SM, Seabra AB, Carvalheira JB, de Oliveira MG, Velloso LA, Curi R \& Saad MJ 2005 S-nitrosation of the insulin receptor, insulin receptor substrate 1 , and protein kinase B/ Akt: a novel mechanism of insulin resistance. Diabetes 54 959-967. (https://doi.org/10.2337/diabetes.54.4.959)

Dong K, Li H, Zhang M, Jiang S, Chen S, Zhou J, Dai Z, Fang Q \& Jia W 2015 Endoplasmic reticulum stress induces up-regulation of hepatic $\beta$-klotho expression through ATF4 signaling pathway. Biochemical \& Biophysical Research Communications 459 300-305. (https://doi. org/10.1016/j.bbrc.2015.02.104)

Fujimoto M, Shimizu N, Kunii K, Martyn JA, Ueki K \& Kaneki M 2005 A role for iNOS in fasting hyperglycemia and impaired insulin signaling in the liver of obese diabetic mice. Diabetes 54 1340-1348. (https:// doi.org/10.2337/diabetes.54.5.1340)

Gomez-Valades AG, Mendez-Lucas A, Vidal-Alabro A, Blasco FX, Chillon M, Bartrons R, Bermudez J \& Perales JC 2008 Pck1 gene silencing in the liver improves glycemia control, insulin sensitivity, and dyslipidemia in db/db mice. Diabetes 57 2199-2210. (https://doi. org/10.2337/db07-1087)

Gross B, Pawlak M, Lefebvre P \& Staels B 2017 PPARs in obesity-induced T2DM, dyslipidaemia and NAFLD. Nature Reviews. Endocrinology 13 36-49. (https://doi.org/10.1038/nrendo.2016.135)

Gum RJ, Gaede LL, Heindel MA, Waring JF, Trevillyan JM, Zinker BA, Stark ME, Wilcox D, Jirousek MR, Rondinone CM, et al. 2003 Antisense protein tyrosine phosphatase 1B reverses activation of p38 mitogen-activated protein kinase in liver of ob/ob mice. Molecular Endocrinology 17 1131-1143. (https://doi.org/10.1210/me.2002-0288)

Hemi R, Yochananov Y, Barhod E, Kasher-Meron M, Karasik A, Tirosh A \& Kanety H 2011 p38 mitogen-activated protein kinase-dependent transactivation of ErbB receptor family: a novel common mechanism for stress-induced IRS-1 serine phosphorylation and insulin resistance. Diabetes 60 1134-1145. (https://doi.org/10.2337/db09-1323)

Jaffrey SR, Snowman AM, Eliasson MJ, Cohen NA \& Snyder SH 1998 CAPON: a protein associated with neuronal nitric oxide synthase that regulates its interactions with PSD95. Neuron 20 115-124. (https:// doi.org/10.1016/s0896-6273(00)80439-0)

Kajiwara A, Tsuchiya Y, Takata T, Nyunoya M, Nozaki N, Ihara H \& Watanabe Y 2013 Nitric oxide enhances increase in cytosolic $\mathrm{Ca}(2+)$ and promotes nicotine-triggered MAPK pathway in PC12 cells. Nitric Oxide : Biology and Chemistry 34 3-9. (https://doi.org/10.1016/j. niox.2013.04.002)

Kohjima M, Enjoji M, Higuchi N, Kato M, Kotoh K, Yoshimoto T, Fujino T, Yada M, Yada R, Harada N, et al. 2007 Re-evaluation of fatty acid metabolism-related gene expression in nonalcoholic fatty liver disease. International Journal of Molecular Medicine 20 351-358. (https://doi.org/10.3892/ijmm.20.3.351)

Lai TW, Zhang S \& Wang YT 2014 Excitotoxicity and stroke: identifying novel targets for neuroprotection. Progress in Neurobiology 115 157-188. (https://doi.org/10.1016/j.pneurobio.2013.11.006)

Li H, Fang Q, Gao F, Fan J, Zhou J, Wang X, Zhang H, Pan X, Bao Y, Xiang K, et al. 2010 Fibroblast growth factor 21 levels are increased https://joe.bioscientifica.com

https://doi.org/10.1530/JOE-20-0322 (c) 2021 Society for Endocrinology Published by Bioscientifica Ltd. Printed in Great Britain 
in nonalcoholic fatty liver disease patients and are correlated with hepatic triglyceride. Journal of Hepatology 53 934-940. (https://doi. org/10.1016/j.jhep.2010.05.018)

Li Q, Zhao Q, Zhang J, Zhou L, Zhang W, Chua B, Chen Y, Xu L \& Li P 2019 The protein phosphatase 1 complex is a direct target of AKT that links insulin signaling to hepatic glycogen deposition. Cell Reports 28 3406-3422.e7. (https://doi.org/10.1016/j. celrep.2019.08.066)

Loria P, Lonardo A \& Anania F 2013 Liver and diabetes. A vicious circle. Hepatology Research 43 51-64. (https://doi.org/10.1111/j.1872034X.2012.01031.x)

Lu M, Wan M, Leavens KF, Chu Q, Monks BR, Fernandez S, Ahima RS, Ueki K, Kahn CR \& Birnbaum MJ 2012 Insulin regulates liver metabolism in vivo in the absence of hepatic Akt and FoxO1. Nature Medicine 18 388-395. (https://doi.org/10.1038/nm.2686)

Lu XM, Zhao H \& Zhang HP 2013 Role of neuronal nitric oxide synthase in the cardiac ischemia reperfusion in mice. Chinese Journal of Physiology 56 291-297. (https://doi.org/10.4077/ CJP.2013.BAB143)

Marsollier N, Kassis N, Mezghenna K, Soty M, Fioramonti X, Lacombe A, Joly A, Pillot B, Zitoun C, Vilar J, et al. 2009 Deregulation of hepatic insulin sensitivity induced by central lipid infusion in rats is mediated by nitric oxide. PLOS ONE 4 e6649. (https://doi.org/10.1371/journal. pone.0006649).

Mezghenna K, Leroy J, Azay-Milhau J, Tousch D, Castex F, Gervais S, Delgado-Betancourt V, Gross R \& Lajoix AD 2014 Counteracting neuronal nitric oxide synthase proteasomal degradation improves glucose transport in insulin-resistant skeletal muscle from Zucker fa/ fa rats. Diabetologia 57 177-186. (https://doi.org/10.1007/s00125-0133084-9)

Mezghenna K, Pomies P, Chalancon A, Castex F, Leroy J, Niclauss N, Nadal B, Cambier L, Cazevieille C, Petit P, et al. 2011 Increased neuronal nitric oxide synthase dimerisation is involved in rat and human pancreatic beta cell hyperactivity in obesity. Diabetologia $\mathbf{5 4}$ 2856-2866. (https://doi.org/10.1007/s00125-011-2264-8)

Millar JS, Stone SJ, Tietge UJ, Tow B, Billheimer JT, Wong JS, Hamilton RL, Farese RV Jr \& Rader DJ 2006 Short-term overexpression of DGAT1 or DGAT2 increases hepatic triglyceride but not VLDL triglyceride or apoB production. Journal of Lipid Research 47 2297-2305. (https://doi.org/10.1194/jlr.M600213JLR200)

Moran-Salvador E, Lopez-Parra M, Garcia-Alonso V, Titos E, MartinezClemente M, Gonzalez-Periz A, Lopez-Vicario C, Barak Y, Arroyo V \& Claria J 2011 Role for PPARgamma in obesity-induced hepatic steatosis as determined by hepatocyte- and macrophage-specific conditional knockouts. FASEB Journal 25 2538-2550. (https://doi. org/10.1096/fj.10-173716)

Mu K, Sun Y, Zhao Y, Zhao T, Li Q, Zhang M, Li H, Zhang R, Hu C, Wang C, et al. 2019 Hepatic nitric oxide synthase 1 adaptor protein regulates glucose homeostasis and hepatic insulin sensitivity in obese mice depending on its PDZ binding domain. EBiomedicine $\mathbf{4 7}$ 352-364. (https://doi.org/10.1016/j.ebiom.2019.08.033)

Muniyappa R, Montagnani M, Koh KK \& Quon MJ 2007 Cardiovascular actions of insulin. Endocrine Reviews 28 463-491. (https://doi. org/10.1210/er.2007-0006)

Nakata S, Tsutsui M, Shimokawa H, Suda O, Morishita T, Shibata K, Yatera Y, Sabanai K, Tanimoto A, Nagasaki M, et al. 2008 Spontaneous myocardial infarction in mice lacking all nitric oxide synthase isoforms. Circulation 117 2211-2223. (https://doi.org/10.1161/ CIRCULATIONAHA.107.742692)
O-Sullivan I, Zhang W, Wasserman DH, Liew CW, Liu J, Paik J, DePinho RA, Stolz DB, Kahn CR, Schwartz MW, et al. 2015 FoxO1 integrates direct and indirect effects of insulin on hepatic glucose production and glucose utilization. Nature Communications 67079 . (https://doi.org/10.1038/ncomms8079)

Pasarin M, Abraldes JG, Rodriguez-Vilarrupla A, La Mura V, GarciaPagan JC \& Bosch J 2011 Insulin resistance and liver microcirculation in a rat model of early NAFLD. Journal of Hepatology 55 1095-1102. (https://doi.org/10.1016/j.jhep.2011.01.053)

Petersen MC \& Shulman GI 2018 Mechanisms of insulin action and insulin resistance. Physiological Reviews 98 2133-2223. (https://doi. org/10.1152/physrev.00063.2017)

Pettinelli P \& Videla LA 2011 Up-regulation of PPAR-gamma mRNA expression in the liver of obese patients: an additional reinforcing lipogenic mechanism to SREBP-1c induction. Journal of Clinical Endocrinology and Metabolism 96 1424-1430. (https://doi.org/10.1210/ jc.2010-2129)

Qu S, Altomonte J, Perdomo G, He J, Fan Y, Kamagate A, Meseck M \& Dong HH 2006 Aberrant Forkhead box O1 function is associated with impaired hepatic metabolism. Endocrinology 147 5641-5652. (https:// doi.org/10.1210/en.2006-0541)

Sansbury BE \& Hill BG 2014 Regulation of obesity and insulin resistance by nitric oxide. Free Radical Biology \& Medicine 73 383-399. (https:// doi.org/10.1016/j.freeradbiomed.2014.05.016)

Shankar RR, Wu Y, Shen HQ, Zhu JS \& Baron AD 2000 Mice with gene disruption of both endothelial and neuronal nitric oxide synthase exhibit insulin resistance. Diabetes 49 684-687. (https://doi. org/10.2337/diabetes.49.5.684)

Sheldon RD, Laughlin MH \& Rector RS 2014 Reduced hepatic eNOS phosphorylation is associated with NAFLD and type 2 diabetes progression and is prevented by daily exercise in hyperphagic OLETF rats. Journal of Applied Physiology 116 1156-1164. (https://doi. org/10.1152/japplphysiol.01275.2013)

Shinozaki S, Choi CS, Shimizu N, Yamada M, Kim M, Zhang T, Shiota G, Dong HH, Kim YB \& Kaneki M 2011 Liver-specific inducible nitric-oxide synthase expression is sufficient to cause hepatic insulin resistance and mild hyperglycemia in mice. Journal of Biological Chemistry $\mathbf{2 8 6}$ 34959-34975. (https://doi.org/10.1074/jbc.M110.187666)

Sun H, Wang X, Chen J, Song K, Gusdon AM, Li L, Bu L \& Qu S 2016 Melatonin improves non-alcoholic fatty liver disease via MAPK-JNK/ P38 signaling in high-fat-diet-induced obese mice. Lipids in Health \& Disease 15 202. (https://doi.org/10.1186/s12944-016-0370-9)

Tateya S, Rizzo NO, Handa P, Cheng AM, Morgan-Stevenson V, Daum G, Clowes AW, Morton GJ, Schwartz MW \& Kim F 2011 Endothelial NO/ cGMP/VASP signaling attenuates Kupffer cell activation and hepatic insulin resistance induced by high-fat feeding. Diabetes $\mathbf{6 0}$ 2792-2801. (https://doi.org/10.2337/db11-0255)

Yang L, Calay ES, Fan J, Arduini A, Kunz RC, Gygi SP, Yalcin A, Fu S \& Hotamisligil GS 2015 METABOLISM. S-Nitrosylation links obesityassociated inflammation to endoplasmic reticulum dysfunction. Science 349 500-506. (https://doi.org/10.1126/science.aaa0079)

Zhang W, Sun Q, Zhong W, Sun X \& Zhou Z 2016 Hepatic peroxisome proliferator-activated receptor gamma signaling contributes to alcohol-induced hepatic steatosis and inflammation in mice. Alcoholism, Clinical and Experimental Research 40 988-999. (https:// doi.org/10.1111/acer.13049)

Zhu LJ, Li TY, Luo CX, Jiang N, Chang L, Lin YH, Zhou HH, Chen C, Zhang Y, Lu W, et al. 2014 CAPON-nNOS coupling can serve as a target for developing new anxiolytics. Nature Medicine 20 1050-1054. (https://doi.org/10.1038/nm.3644)

Received in final form 26 November 2020

Accepted 5 January 2021

Accepted Manuscript published online 8 January 2021 https://joe.bioscientifica.com https://doi.org/10.1530/JOE-20-0322 (c) 2021 Society for Endocrinology Published by Bioscientifica Ltd. Printed in Great Britain 